Pure and Applied Mathematics Quarterly

Volume 6, Number 4

(Special Issue: In honor of

Joseph J. Kohn, Part 2 of 2$)$

$1035-1050,2010$

\title{
Higher Order Invariants of Levi Degenerate Hypersurfaces
}

\author{
Martin Kolář \\ Dedicated to Professor J.J.Kohn on the occasion of his 75th birthday
}

\begin{abstract}
The first part of this paper considers higher order CR invariants of three dimensional hypersurfaces of finite type. Using a full normal form we give a complete characterization of hypersurfaces with trivial local automorphism group, and analogous results for finite groups. The second part considers hypersurfaces of finite Catlin multitype, and the Kohn-Nirenberg phenomenon in higher dimensions. We give a necessary condition for local convexifiability of a class of pseudoconvex hypersurfaces in $\mathbb{C}^{n+1}$.
\end{abstract}

Keywords: Local automorphism group, finite type hypersurface, KohnNirenberg invariants, Catlin multitype, normal form.

\section{INTRODUCTION}

In complex dimension two, the lowest order local CR invariant of a smooth hypersurfaces $M \subseteq \mathbb{C}^{2}$ at a point $p \in M$ is the type of the point. This fundamental invariant was introduced by J. J. Kohn in [9], in order to study boundary behavior of the $\bar{\partial}$ operator on weakly pseudoconvex domains. It measures the number of commutators of $\mathrm{CR}$ and anti-CR vector fields needed to span the whole complexified tangent space to $M$ at $p$. In more geometric terms, the type of $p$ is the maximal order of contact between $M$ and complex curves passing through $p$.

Received December 7, 2007.

The author was supported by a grant of the GA ČR no. 201/05/2117 
On the next level, there is a well defined model hypersurface at $p$, which gives important numerical local invariants, characterizing local geometry of $M$ ([10]).

In higher dimensions, local geometry of Levi degenerate hypersurfaces is much more complicated. The order of contact with complex curves does not give an open condition anymore, and cannot characterize subellipticity of the $\bar{\partial}$ operator on the boundary. In order to obtain invariants relevant for analysis of the $\bar{\partial}$ equation, one has to consider orders of contact with singular complex varieties. Such invariants have been introduced in the work of J. P. D'Angelo ([5]).

Let $M$ be a smooth hypersurface in $\mathbb{C}^{n+1}$, where $n \geq 2$. If $d_{k}$ denotes the maximal order of contact of $M$ with complex varieties of dimension $k$ at the given point, the n-tuple $\left(d_{n}, \ldots, d_{1}\right)$ is called the D'Angelo multitype of $p$.

For pseudoconvex hypersurfaces, D. Catlin ([3]) introduced a different, more algebraic notion of multitype. As an important advantage, it provides a well defined weighted-homogeneous model, an essential tool for local analysis. The two notions of multitype coincide on a class of hypersurfaces called semiregular ([6]), or h-extendible ([20]). It contains, for example, all decoupled and all convexifiable hypersurfaces.

Since the definition of multitype is nonconstructive, and the models are not uniquely defined, it is not a priori clear what is the relation between various models. In some situations, when low order boundary invariants are needed, it is enough to choose an arbitrary model. On the other hand, in order to study higher order CR invariants it is essential to understand the non-uniqueness in the definition of models. In particular, it is not a priori obvious whether all models are necessarily biholomorphically equivalent. In the case of h-extendible pseudoconvex hypersurfaces, biholomorphic equivalence of models was proved by N. Nikolov in [19].

The first part of this paper considers local CR invariants of hypersurfaces of finite type in $\mathbb{C}^{2}$. In dimension two, a complete set of local $\mathrm{CR}$ invariants can be constructed, on the level of formal power series, using analysis of generalized Chern-Moser operators ([15]). Combined with the result of M. S. Baouendi, P. Ebenfelt and L. P. Rothschild on convergence of formal equivalences, it gives a solution to the local equivalence problem. One of the main applications of this result provides understanding of local symmetries of finite type hypersurfaces. 
In Section 3 we give a characterization of finite type hypersurfaces with trivial local automorphism group, in terms of normal form coordinates. Analogous complete classification is obtain in the case of finite local automorphism group of order $m>1$.

In Section 4 we consider a constructive approach to the Catlin multitype on a general (not necessarily pseudoconvex) hypersurface in $\mathbb{C}^{n+1}$. Section 5 gives a review of Kohn-Nirenberg invariants in complex dimension two. The results are used in Section 6, where we define certain numerical invariants on hypersurfaces for which at the given point all multitype entries coincide. We show how they can be used to study the Kohn-Nirenberg phenomenon in higher dimensions. In particular, we obtain a necessary condition for local convexifiability of such hypersurfaces.

\section{Preliminaries}

In the first part of this paper, we will consider a real analytic hypersurface $M \subseteq \mathbb{C}^{2}$ and a Levi degenerate point $p \in M$ of finite type $k>2$.

In order to describe $M$ in a neighbourhood of $p$, we will use local holomorphic coordinates $(z, w)$ centered at $p$, where $z=x+i y, w=u+i v$. The hyperplane $\{v=0\}$ will be assumed tangent to $M$ at $p$. In this setting, $M$ is described near $p$ as the graph of a uniquely determined real valued function

$$
v=\Psi(z, \bar{z}, u) .
$$

Recall that $p \in M$ is a point of finite type $k$ if and only if there exist local holomorphic coordinates such that $M$ is given by

$$
v=P(z, \bar{z})+o\left(|z|^{k}+|u|\right),
$$

where the leading term is a nonzero real valued homogeneous polynomial of degree $k$ without harmonic terms,

$$
P(z, \bar{z})=\sum_{j=e}^{k-e} a_{j} z^{j} \bar{z}^{k-j},
$$

with $1 \leq e \leq \frac{k}{2}$ and

$$
a_{e}=1 \text {. }
$$

We will now recall some basic facts from the normal form construction [15]. 
Using (2), we will define two basic integer valued invariants. The first one, denoted by $e$ and defined implicitly by (2), is the essential type of the model hypersurface (defined below).

When $e<\frac{k}{2}$, the second invariant is defined as follows. Let $e=m_{0}<m_{1}<$ $\cdots<m_{s}<\frac{k}{2}$ be the indices in (2) for which $a_{m_{i}} \neq 0$. The invariant, denoted by $d$, is the greatest common divisor of the numbers $k-2 m_{0}, k-2 m_{1}, \ldots, k-2 m_{s}$.

The polynomial $P$ need not be determined uniquely by (2), (3). In order to make it unique, the following condition is imposed. Denote

$$
q_{i}=\frac{\operatorname{gcd}\left(k-2 m_{0}, k-2 m_{1}, \ldots, k-2 m_{i}\right)}{\operatorname{gcd}\left(k-2 m_{0}, k-2 m_{1}, \ldots, k-2 m_{i+1}\right)}
$$

for $0 \leq i \leq s-1$. In addition to (2), (3), we require that $P$ satisfies

$$
\arg a_{m_{i+1}} \in\left[0, \frac{2 \pi}{q_{i}}\right)
$$

for $0 \leq i \leq s-1$. This determines $P$ uniquely.

The model hypersurface $M_{H}$ to $M$ at $p$ is defined using the normalized leading homogeneous term,

$$
M_{H}=\left\{(z, w) \in \mathbb{C}^{2} \mid v=\sum_{j=e}^{k-e} a_{j} z^{j} \bar{z}^{k-j}\right\} .
$$

In particular, when the leading term is circular, we write

$$
S_{k}=\left\{\left.(z, w) \in \mathbb{C}^{2}|v=| z\right|^{k}\right\} .
$$

Another exceptional model is the tubular hypersurface

$$
T_{k}=\left\{(z, w) \in \mathbb{C}^{2} \mid v=\frac{1}{k}\left[(z+\bar{z})^{k}-2 \operatorname{Re} z^{k}\right]\right\} .
$$

The local automorphism group of a hypersurface $M$ at a point $p$ will be denoted by $\operatorname{Aut}(M, p)$. It was proved in [15] that if $e<\frac{k}{2}$, the local automorphism group of $M_{H}$ consists of transformations

$$
z^{*}=\delta \exp i \theta z, \quad w^{*}=\delta^{k} w,
$$

where $\exp i \theta$ is a $d$-th root of unity and $\delta>0$ for $k$ even or $\delta \in \mathbb{R} \backslash\{0\}$ for $k$ odd. Hence $\operatorname{Aut}\left(M_{H}, p\right)=\mathbb{R}^{+} \oplus \mathbb{Z}_{d}$ for $k$ even and $\operatorname{Aut}\left(M_{H}, p\right)=\mathbb{R}^{*} \oplus \mathbb{Z}_{d}$ for $k$ odd. 
The local automorphism group of $S_{k}$ is three dimensional, consisting of transformations of the form

$$
f(z, w)=\frac{\delta e^{i \theta} z}{(1+\mu w)^{\frac{1}{e}}}, \quad g(z, w)=\frac{\delta^{k} w}{1+\mu w},
$$

with $\delta>0$, and $\theta, \mu \in \mathbb{R}$.

\section{LocAl AUTOMORPHisM GROUPS IN $\mathbb{C}^{2}$}

We write

$$
\Psi(z, \bar{z}, u)=P(z, \bar{z})+F(z, \bar{z}, u)
$$

where

$$
F(z, \bar{z}, u)=\sum_{j, l, m} a_{j l m} z^{j} \bar{z}^{l} u^{m}
$$

We will also consider the Taylor expansion of $F$ in terms of $z, \bar{z}$,

$$
F(z, \bar{z}, u)=\sum_{j, l} F_{j l}(u) z^{j} \bar{z}^{l}
$$

where

$$
F_{j l}(u)=\sum_{m} a_{j l m} u^{m} .
$$

The results of [15] give three different complete normal forms, depending on the form of the model. There are two exceptional models, $S_{k}$ and $T_{k}$, while the generic case covers all remaining models.

When $e=\frac{k}{2}$, we have the following complete normalization. $F$ is in normal form if

$$
\begin{aligned}
F_{j 0} & =0, \quad j=0,1, \ldots, \\
F_{e, e+j} & =0, \quad j=0,1, \ldots, \\
F_{2 e, 2 e} & =0, \\
F_{3 e, 3 e} & =0, \\
F_{2 e, 2 e-1} & =0 .
\end{aligned}
$$

Normal coordinates (i.e. such in which the normal form conditions hold), are determined uniquely up to the action of the symmetry group (8). 
When $M_{H}=T_{k}$, we have the following normal form conditions:

$$
\begin{aligned}
F_{j 0}=0, \quad j=1,2, \ldots, \\
F_{k-1+j, 1}=0, \quad j=0,1, \ldots,
\end{aligned}
$$

and

$$
F_{2 k-2,2}=\operatorname{Re} F_{k-2,1}=\operatorname{Re} F_{k, k-1}=0 .
$$

Again, normal coordinates are determined uniquely up to the action of the symmetry group $\operatorname{Aut}\left(T_{k}, 0\right)$.

Now, let $M_{H}$ be a generic model, i.e. $e<\frac{k}{2}$ and $M_{H}$ is different from $T_{k}$. Denote $F_{k-1}(u)=\left(F_{1, k-2}(u), F_{2, k-3}(u), \ldots, F_{k-2,1}(u)\right)$. The normal form conditions are:

$$
\begin{aligned}
F_{j 0} & =0, \quad j=1,2, \ldots, \\
F_{k-e+j, e} & =0, \quad j=0,1, \ldots, \\
F_{2 k-2 e, 2 e} & =0, \\
\left(F_{k-1}, P_{z}\right) & =0,
\end{aligned}
$$

where

$$
\left(F_{k-1}, P_{z}\right)=\sum_{j=1}^{k-2} F_{j, k-1-j}(j+1) \bar{a}_{j+1} .
$$

The corresponding normal coordinates are unique up to the action of the symmetry group $\operatorname{Aut}\left(M_{H}, 0\right)$.

The following result was obtained in [16].

Proposition 3.1. Let $M$ be a Levi degenerate hypersurface of finite type with $e=\frac{k}{2}$, not equivalent to $v=|z|^{k}$. Then all local automorphisms expressed in normal coordinates have the form of decoupled linear transformations

$$
z^{*}=\delta e^{i \theta} z, \quad w^{*}=\delta^{k} w,
$$

for some $\theta \in \mathbb{R}$ and $\delta \in \mathbb{R}^{*}$.

Now we turn to the general case. Let $(z, w)$ be normal coordinates for $M$ at $p$, and $v=P(z, \bar{z})+F(z, \bar{z}, u)$ be the defining equation in such coordinates. Denote

$$
\Theta_{1}=\left\{(j, l, m) \in \mathbb{Z}_{+}^{3}: j \neq l \text { and } a_{j, l, m} \neq 0\right\}
$$


and

$$
\Theta_{2}=\left\{(j, k-j, 0) \in \mathbb{Z}_{+}^{3}: j \neq k-j \text { and } a_{j} \neq 0\right\} .
$$

Set $\Theta=\Theta_{1} \cup \Theta_{2}$. If $\Theta$ is nonempty, we define

$$
\mu_{0}=\operatorname{gcd}_{(j, l, m) \in \Theta}|j-l| .
$$

We have the following complete description of all finite type hypersurfaces with finite local automorphism group.

Theorem 3.1. $\quad$ Let $M$ be a Levi degenerate hypersurface of finite type, not equivalent to a model hypersurface. Then $\operatorname{Aut}(M, p)$ is finite if and only if $\Theta$ is nonempty. In this case, the stability group is isomorphic to $\mathbb{Z}_{\mu_{0}}$.

proof: First we will prove that in normal coordinates all local automorphisms of $M$ are decoupled linear, of the form (14). By Proposition 3.1, it remains to consider the case $e<\frac{k}{2}$.

By definition, any local automorphism of $M$ in normal coordinates preserves normal form. In [15], such transformations are completely characterized, and correspond to the action of the local symmetry group of the model.

Starting with the generic case, we assume $M_{H}$ is a generic model, and consider the normal form conditions (12). The local symmetry group of the model acts on normal forms. We will prove that the transformation by each element of $\operatorname{Aut}\left(M_{H}, p\right)$ preserves the normal form, hence its action on normal forms is direct and no renormalization is needed. Since every element of $\operatorname{Aut}\left(M_{H}, p\right)$ is a decoupled linear transformation, its application clearly preserves the first three conditions. In order to see that the last condition is also preserved, we write $P$ as

$$
P(z, \bar{z})=\sum_{j=1}^{k-1} a_{j} z^{j} \bar{z}^{k-j},
$$

where $a_{j} \neq 0$ only if $k-2 j$ is divisible by $d$. Denote

$$
\beta_{j}(u)=F_{j, k-1-j}(u) .
$$

We have

$$
P_{z}(z, \bar{z})=\sum_{j=1}^{k-1} j a_{j} z^{j-1} \bar{z}^{k-j}
$$


Since dilations clearly preserve all the normal form conditions, we consider the action of a transformation $z^{*}=\alpha z$, where $\alpha^{d}=1$. Using this and the fact that $a_{j} \neq 0$ implies $k-2 j$ is divisible by $d$, we obtain

$$
\begin{gathered}
\left(F_{k-1}^{*}, P_{z}\right)=\sum_{j=2}^{k-1} j \bar{a}_{j} \beta_{j-1}(u) \alpha^{j-1} \bar{\alpha}^{k-j}= \\
=\alpha^{-1} \sum_{j=2}^{k-1} j \bar{a}_{j} \beta_{j-1}(u) \alpha^{j} \bar{\alpha}^{k-j}=\alpha^{-1}\left(F_{k-1}, P_{z}\right)=0,
\end{gathered}
$$

which proves the claim. Now let the model be the tubular hypersurface $T_{k}$. In this case $e=1$, and since all the coefficients $a_{j}$ of $P$ are nonzero, we obtain immediately the value of $d$. If $k$ is even, all the numbers $k-2 m_{0}, k-2 m_{1}, \ldots, k-$ $2 m_{s}$ are even, and we have $d=2$. If $k$ is odd, then again immediately $d=1$.

Consider the normal form conditions (10), (11). Clearly this normalization is preserved when an element of $A u t\left(T_{k}, 0\right)$ is applied.

Thus we have proved that whenever $M$ is different from $S_{k}$, all local automorphisms in normal coordinates are of the form (14). Hence it remains to consider the action of (14) on the defining equation of $M$.

If $\Theta$ is empty, then any rotation in $z$ preserves $M$, so the local automorphism group is infinite. Let us assume now that $\Theta$ is not empty.

Since all local automorphisms are decoupled linear in normal coordinates, they act on each monomial in the expansion of $F$ separately, in an obvious way. Since $M$ is not a model, it follows immediately that weighted dilations do not preserve $F$. If $(j, l, m) \in \Theta$, then the coefficient $a_{j, l, m}$ is preserved by a rotation $z^{*}=$ $\exp i \theta z$ if and only if

$$
(\exp i \theta)^{j-l}=1 .
$$

Since this holds for any element of $\Theta$, the result follows.

As a particular case, we obtain a complete description of hypersurfaces with trivial local automorphism group.

Theorem 3.2. $\quad$ Let $M$ be a Levi degenerate hypersurface of finite type, not equivalent to a model. Aut $(M, p)$ is trivial if and only if $\Theta$ is nonempty and $\mu_{0}=1$. 


\section{Hypersurfaces of Finite multitype}

Let $M \subseteq \mathbb{C}^{n+1}$ be a smooth hypersurface (not necessarily pseudoconvex), and $p$ be a Levi degenerate point on $M$. We will assume that $p$ is a point of finite type in the sense of Bloom and Graham. In this section we consider a constructive approach to the Catlin's definition of multitype.

Consider local holomorphic coordinates $(z, w)$, where $z=\left(z_{1}, z_{2}, \ldots, z_{n}\right)$ and $w=u+i v, z_{j}=x_{j}+i y_{j}$, centered at the point $p \in M$. Again, the hyperplane $\{v=0\}$ is assumed to be tangent to $M$ at $p . M$ is described near $p$ as the graph of a uniquely determined real valued function

$$
v=\Psi\left(z_{1}, \ldots, z_{n}, \bar{z}_{1}, \ldots, \bar{z}_{n}, u\right) .
$$

We now apply Catlin's definition of multitype to $M$ at $p$. In the following, $\alpha, \beta$ will denote multiindices, and we will use the standard multiindex notation.

Definition 4.1. A weight is an n-tuple of nonnegative rational numbers $\Lambda=$ $\left(\lambda_{1}, \ldots, \lambda_{n}\right)$, where $0 \leq \lambda_{j} \leq \frac{1}{2}$, and $\lambda_{j} \geq \lambda_{j+1}$, such that for each $k$ either $\lambda_{k}=0$, or there exist nonnegative integers $l_{1}, \ldots, l_{k}$ satisfying $l_{k}>0$ and

$$
\sum_{j=1}^{k} l_{j} \lambda_{j}=1
$$

The component $\lambda_{j}$ of $\Lambda$ is interpreted as the weight of the variable $z_{j}$. The variables $w$ and $u$ are given weight one. The weighted degree of a monomial $c_{\alpha \beta l} z^{\alpha} \bar{z}^{\beta} u^{l}$ is

$$
w t\left(c_{\alpha \beta l} z^{\alpha} \bar{z}^{\beta} u^{l}\right)=l+\sum_{i=1}^{n}\left(\alpha_{i}+\beta_{i}\right) \lambda_{i}
$$

A real valued polynomial $P(z, \bar{z}, u)$ is $\Lambda$ - homogeneous of weighted degree $\kappa$ if it is a sum of monomials of weight $\kappa$.

A weight $\Lambda$ will be called distinguished if there exist coordinates $(z, w)$ in which the defining equation has form

$$
v=P(z, \bar{z})+o_{w t}(1)
$$


where $P(z, \bar{z})$ is a $\Lambda$ - homogeneous polynomial of weighted degree one which is not pluriharmonic, and $o_{w t}(1)$ denotes terms in the Taylor expansion of weight greater than one.

The fact that distinguished weights do exist follows from the assumption of Bloom-Graham finite type ([2]).

We denote by $\Lambda_{M}=\left(\mu_{1}, \ldots, \mu_{n}\right)$ the infimum of distinguished weights with respect to the lexicographic ordering.

The multitype of $M$ at $p$ is defined to be the n-tuple $\left(m_{1}, m_{2}, \ldots, m_{n}\right)$, where $m_{j}=\frac{1}{\mu_{j}}$ if $\mu_{j} \neq 0$ and $m_{j}=\infty$ if $\mu_{j}=0$. If none of the $m_{j}$ is infinity, we say that $M$ is of finite multitype at $p$.

Note that since the definition of multitype considers all distinguished weights, the infimum is a biholomorphic invariant, and we may speak of the multitype.

Coordinates corresponding to a distinguished weight $\Lambda$, in which the local description of $M$ has form (16), with $P$ being $\Lambda$ - homogeneous, will be called $\Lambda$ - adapted.

$\Lambda_{M}$ will be called the multitype weight. Note that for any $\delta>0$ there exist only finitely many points $\left(l_{1}, \ldots, l_{n}\right) \in \mathbb{Z}_{+}^{n}$ such that $l_{j} \leq \frac{1}{\delta}$ for all $j=1, \ldots, n$. It follows immediately that if $M$ at $p$ is of finite multitype, $\Lambda_{M}$ - adapted coordinates do exist (cf. [3]).

From now on we assume that $p \in M$ is of finite multitype. If (16) is the defining equation in some $\Lambda_{M}$ - adapted coordinates, we define a model hypersurface to $M$ at $p$ to be

$$
M_{H}=\left\{(z, w) \in \mathbb{C}^{n+1} \mid v=P(z, \bar{z})\right\}
$$

Models are useful for many geometric and analytic results. In order to deal with biholomorphisms between models, we introduce the following terminology. Here weighted degree is understood with respect to the multitype weight $\Lambda_{M}$.

Definition 4.2. A transformation

$$
w^{*}=w+g\left(z_{1}, \ldots z_{n}, w\right), \quad z_{i}^{*}=z_{i}+f_{i}\left(z_{1}, \ldots z_{n}, w\right)
$$


preserving form (15) is called

- homogeneous if $f_{i}$ is a $\Lambda_{M}$-homogeneous polynomial of weighted degree $\mu_{i}$ and $g$ is a $\Lambda_{M}$-homogeneous polynomial of weighted degree one,

- subhomogeneous if $f_{i}$ is a polynomial consisting of monomials of weighted degree less or equal to $\mu_{i}$ and $g$ consists of monomials of weighted degree less or equal to one,

- superhomogeneous if the Taylor expansion of $f_{i}$ consists of terms of weighted degree greater or equal to $\mu_{i}$ and $g$ consists of terms of weighted degree greater or equal to one.

We write $P$ in the form

$$
P(z, \bar{z})=\sum_{\left|\alpha, \alpha^{\prime}\right|_{\Lambda_{M}}=1} A_{\alpha, \alpha^{\prime}} z^{\alpha} \bar{z}^{\alpha^{\prime}}
$$

where $\left|\alpha, \alpha^{\prime}\right|_{\Lambda_{M}}=\sum_{j=1}^{n} \mu_{j}\left(\alpha_{j}+\alpha^{\prime}{ }_{j}\right)$. Homogeneous transformations are of the form

$$
z_{i}^{*}=z_{i}+\sum_{|\alpha|_{\Lambda_{M}}=\mu_{i}} C_{\alpha} z^{\alpha}, \quad w^{*}=c w+\sum_{|\alpha|_{\Lambda_{M}}=1} D_{\alpha} z^{\alpha}
$$

where $|\alpha|_{\Lambda_{M}}=\sum_{j=1}^{n} \mu_{j} \alpha_{j}$ and $c \in \mathbb{R}^{*}$.

Let us remark that the problem of biholomorphic equivalence of models is considered in [14]. The following result was obtained there. Note that models are understood in the sense of this section, i.e. corresponding to $\Lambda_{M}$-adapted coordinates.

Theorem 4.1. A biholomorphic transformation takes $\Lambda_{M}$-adapted coordinates into $\Lambda_{M}$-adapted coordinates if and only if it is superhomogeneous. Moreover, let $M_{H}$ and $\tilde{M}_{H}$ be two models for $M$ at $p$. Then there is a homogeneous transformation which maps $M_{H}$ to $\tilde{M}_{H}$. In particular, any two models are biholomorphic by a polynomial transformation. 


\section{KOHN-NIRENBERG HYPERSURFACES}

We now review some explicit conditions for local convexifiability of pseudoconvex hypersurfaces in complex dimension two, which will be used in the next section. There are no new results in this section.

Let

$$
P(z, \bar{z})=a_{0}|z|^{k}+\sum_{j=2,4, \ldots, k}|z|^{k-j} \operatorname{Re}\left(a_{j} z^{j}\right)
$$

be a subharmonic but not harmonic homogeneous polynomial of degree $k$. We will denote

$$
\gamma_{l k}=\frac{k}{l^{2}-k}
$$

if $l^{2} \geq 3 k-2$ and

$$
\gamma_{l k}=\sqrt{\frac{\left(4 k-l^{2}-4\right) k^{2}}{(4 k-4)\left(k^{2}-l^{2}\right)}}
$$

if $l^{2} \leq 3 k-2$.

Further, we consider hypersurfaces of the Kohn-Nirenberg type. Let

$$
M_{a}^{k, l}=\left\{(z, w) \in \mathbb{C}^{2} \mid \operatorname{Im} w=P_{a}^{k, l}(z, \bar{z})\right\},
$$

where

$$
P_{a}^{k, l}(z, \bar{z})=|z|^{k}+a|z|^{k-l} \operatorname{Re} z^{l}
$$

with $a \geq 0$.

We have the following characterization of convexifiability of $M_{a}^{k, l}$, obtained in [11].

Proposition 5.1. $M_{a}^{k, l}$ is convex if and only if $a \leq \gamma_{l k}$. Moreover, if $l$ is not a divisor of $k$, then this condition is equivalent to convexifiability of $M_{a}^{k, l}$.

The following result appears in [13], as Theorem 3 .

Proposition 5.2. Let the model at $p \in M$ be given by (20). If $M$ is convexifiable at $p$, then

(i) $\frac{\left|a_{j}\right|}{a_{0}} \leq \gamma_{j k}$ for all $j>\frac{k}{2}$,

and

(ii) $\frac{\left|a_{j}\right|}{a_{0}} \leq 2 \gamma_{j k}$ for all $j \leq \frac{k}{2}$. 


\section{Hypersurfaces With homogeneous models}

We will now consider a smooth pseudoconvex hypersurface $M \subseteq \mathbb{C}^{n+1}$ and local holomorphic coordinates $\left(z_{1}, z_{2}, \ldots, z_{n}, w\right)$, where $w=u+i v$ and $z_{j}=x_{j}+i y_{j}$, centered at a point $p \in M$. We assume $p$ is of finite Catlin multitype.

Consider $\Lambda_{M}$ - adapted coordinates, in which the hypersurface is described by

$$
v=P\left(z_{1}, \ldots, z_{n}, \bar{z}_{1}, \ldots, \bar{z}_{n}\right)+o_{w t}(1) .
$$

It is well known that on any locally convex domain the Catlin and D'Angelo multitypes coincide, and the numbers $m_{j}$ are all even integers (see [21]). Hence inequality of the two multitypes is a trivial obstruction to convexifiability. Our aim is to study other possible obstructions, hence we restrict ourselves to domains on which the two multitypes coincide. This simplifies substantially the form of the leading polynomial $P(z, \bar{z})$. When restricted to a coordinate axis $z_{j}$ it gives a subharmonic but not harmonic real valued homogeneous polynomial of degree $m_{j}$ of the form

$$
P_{j}\left(z_{j}, \bar{z}_{j}\right)=a_{0}^{j}\left|z_{j}\right|^{m_{j}}+\sum_{i=2,4, \ldots, m_{j}}\left|z_{j}\right|^{m_{j}-i} \operatorname{Re}\left(a_{i}^{j} z_{j}^{i}\right)
$$

for some $a_{i}^{j} \in \mathbb{C}$ and $a_{0}^{j}>0$.

We will now consider hypersurfaces for which the multitype at the given point satisfies $m_{1}=m_{2}=\cdots=m_{n}=m$, each entry being equal to a fixed even integer $m$. Hence, in $\Lambda_{M}$ - adapted coordinates, the leading polynomial $P$ is a plurisubharmonic homogeneous polynomial of degree $m$ which, by the equality of multitypes, is not harmonic along any complex line passing through the origin. Indeed, if it were harmonic along such a line, the order of contact with complex curves would exceed $m$. In this case for all homogeneous and subhomogeneous transformations the $f$ component of the transformation is linear. This substantially simplifies the analysis.

We define the Kohn-Nirenberg numbers of $M$ at $p$ as follows. For a nonzero vector $c=\left(c_{1}, \ldots c_{n}\right) \in \mathbb{C}^{n}$ we consider the restriction of $P$ to the complex line

$$
\Gamma_{c}=\left\{z \in \mathbb{C}^{n} ; z=\zeta c, \zeta \in \mathbb{C}\right\}
$$

generated by $c$. This restriction as a function of $\zeta$ is a subharmonic polynomial of the form (20), which we denote $P_{c}$, and its coefficients by $a_{j}^{c}$. For an even integer 
$l$ we define the Kohn-Nirenberg number

$$
\kappa_{M}^{l}=\sup _{c} \frac{\left|a_{l}^{c}\right|}{a_{0}^{c}} .
$$

We have the following necessary condition for local convexifiability.

Proposition 6.1. If there exists an $l>\frac{m}{2}$ such that $\kappa_{M}^{l}>\gamma_{l m}$, or an $l \leq \frac{m}{2}$ such that $\kappa_{M}^{l}>2 \gamma_{l m}$, then $M$ is not locally convexifiable.

proof: By Proposition 5.2., $M$ is not convex in the original coordinates $(z, w)$. Let $\left(z^{*}, w^{*}\right)$ be another system of local holomorphic coordinates, and let the biholomorphic coordinate change be given by

$$
\begin{aligned}
z_{i}^{*} & =f_{i}(z, w) \\
w^{*} & =g(z, w) .
\end{aligned}
$$

We may restrict attention to transformations which preserve the form (15), and moreover satisfy the normalization condition $g_{w}(0,0)=1$. The general case is obtained from this by an affine transformation, which does not affect convexity.

Let $F^{*}$ denote the function which describes $M$ in the new coordinates. By substituting (23) into $v^{*}=F^{*}\left(z^{*}, \bar{z}^{*}, u^{*}\right)$, we obtain

$$
\begin{aligned}
& F^{*}(f(z, u+i F(z, \bar{z}, u)), \overline{f(z, u+i F(z, \bar{z}, u))}, \\
& \quad \operatorname{Re} g(z, u+i F(z, \bar{z}, u))=\operatorname{Im} g(z, \operatorname{Re} g(z, u+i F(z, \bar{z}, u)),
\end{aligned}
$$

where $f=\left(f_{1}, \ldots, f_{n}\right)$. Now we will distinguish two cases. First, let $g$ contain terms of weight less than one. Let $g_{\kappa}$ denote the leading homogeneous term in $g$, where $\kappa$ denotes the corresponding weight. By comparing terms of weight $\kappa$ in (24), it follows that $F^{*}$ starts with a nonzero pluriharmonic polynomial $Q(z, \bar{z})$ of weight $\kappa$. We choose a complex line $\Gamma_{c}$ of the form (22), such that the restriction of $Q$ to $\Gamma_{c}$ is nonzero. The defining equation restricted to this line has form

$$
v=R e \alpha \zeta^{m \kappa}+o(m \kappa),
$$

where $\alpha \neq 0$, hence $F^{*}$ is not locally convex.

Let now $g$ contain only terms of weight greater or equal to one, and let $g_{1}$ denote the homogeneous part of $g$ of weight one. We separate the leading linear term in $f(z, w)$ in the $z$-variables. Write

$$
f_{i}(z, w)=L_{i}(z)+o_{w t}\left(\frac{1}{m}\right)
$$


and denote $L(z)=\left(L_{1}(z), \ldots, L_{n}(z)\right)$. Let $P^{*}$ denote the leading homogeneous term in $F^{*}$. Since there are no terms of weight less than one on the right hand side of $(24), P^{*}$ is of weight one. For terms of weight one in (24) we obtain

$$
P^{*}(L(z), \overline{L(z)})=P(z, \bar{z})+\operatorname{Im} g_{1}(z) .
$$

It follows that the leading term in $F^{*}$ is obtained from $P$ by a linear transformation in $z$ and addition of pluriharmonic terms. In the original coordinates we choose a line $\Gamma_{c}$, on which $\frac{\left|a_{l}^{c}\right|}{a_{0}^{c}}>\gamma_{l m}$, for some $l>\frac{m}{2}$ or $\frac{\left|a_{l}^{c}\right|}{a_{0}^{c}}>2 \gamma_{l m}$, for some $l \leq \frac{m}{2}$, and denote $\Gamma_{c}^{*}$ the image of this line by the linear part $L(z)$. It follows from linearity that the same condition holds on this line, hence by Proposition $5.2 ., F^{*}$ is not convex.

\section{REFERENCES}

[1] Baouendi, M. S., Ebenfelt, P., Rothschild, L. P., Convergence and finite determination of formal CR mappings, J. Amer. Math. Soc. 13, (2000), 697-723.

[2] Bloom, T. and Graham, I., On "type" conditions for generic real submanifolds of $C^{n}$, Invent. Math. 40 (1977), 217-243.

[3] Catlin, D., Boundary invariants of pseudoconvex domains, Ann. Math. 120 (1984), 529586 .

[4] Chern, S. S. and Moser, J., Real hypersurfaces in complex manifolds, Acta Math. 133 (1974), 219-271.

[5] D'Angelo, J., Orders od contact, real hypersurfaces and applications, Ann. Math. 115 (1982), 615-637.

[6] Diedrich, K. and Herbort, G., Pseudoconvex domains of semiregural type, in Contributions to Complex Analysis and Analytic geometry 127-161 (1994).

[7] Diederich, K. and McNeal, J. D., Pointwise nonisotropic support functions on convex domains, Progress Math. 188 (2000), 184-192.

[8] Ebenfelt, P., Lamel, B., Zaitsev, D., Degenerate real hypersurfaces in $\mathbb{C}^{2}$ with few automorphisms, Trans. Amer. Math. Soc. 361 (2009), 3241-3267.

[9] Kohn, J. J., Boundary behaviour of $\bar{\partial}$ on weakly pseudoconvex manifolds of dimension two, J. Diff. Geom. 6 (1972), 523-542.

[10] Kohn, J. J. and Nirenberg, L., A pseudoconvex domain not admitting a holomorphic support function, Math. Ann. 201 (1973), 265-268.

[11] Kolář, M., Convexifiability and supporting functions in $\mathbb{C}^{2}$, Math. Res. Lett. 2 (1995), 505-513.

[12] Kolár̆, M., Generalized models and local invariants of Kohn-Nirenberg domains, Math. Z., 259 (2008), 277-286. 
[13] Kolár̆, M., Necessary conditions for local convexifiability of pseudoconvex domains in $\mathbb{C}^{2}$, Rend. Circ. Mat. Palermo (2), 69 (2002), 109-116.

[14] Kolár, M., The Catlin multitype and biholomorphic equivalence of models, preprint

[15] Kolář, M., Normal forms for hypersurfaces of finite type in $\mathbb{C}^{2}$, Math. Res. Lett. 12 (2005), 897-910.

[16] Kolář, M., Local symmetries of finite type hypersurfaces in $\mathbb{C}^{2}$, Sci. China A 49 (2006), 1633-1641.

[17] McNeal, J. D., Estimates on the Bergman Kernels on Convex Domains, Adv. Math. 109 (1994), 108-139.

[18] McNeal, J. D., Uniform subelliptic estimates on scaled convex domains of finite type, Proc. Amer. Math. Soc. 130 (2002), 39-47 (electronic).

[19] Nikolov, N., Biholomorphy of the model domains at a semiregular boundary point C. R. Acad. Bulgare Sci. 55 (2002), 5-8.

[20] Yu, J., Peak functions on weakly pseudoconvex domains, Indiana Univ. Math. J. 43 (1994), 1271-1295.

[21] Yu, J., Multitypes of convex domains, Indiana Univ. Math. J. 41 (1992), 837-849.

\section{Martin Kolář}

Department of Mathematics and Statistics

Masaryk University, Kotlarska 2, 61137 Brno

E-mail: mkolar@math.muni.cz 